\title{
Ballet Balance Strategies
}

Pedersen, Camilla; Erleben, Kenny; Sporring, Jon

Published in:

Simulation (San Diego, Calif.)

Publication date:

2006

Document version

Early version, also known as pre-print

Citation for published version (APA):

Pedersen, C., Erleben, K., \& Sporring, J. (2006). Ballet Balance Strategies. Simulation (San Diego, Calif.), 14(8), 1135-1142. 


\title{
Ballet balance strategies
}

\author{
Camilla Pedersen ${ }^{\mathrm{a}}$, Kenny Erleben ${ }^{\mathrm{b}}$, Jon Sporring ${ }^{\mathrm{b}, *}$ \\ ${ }^{a}$ IT University of Copenhagen, Rued Langgaards Vej 7, DK-2300 Copenhagen, Denmark \\ ${ }^{\mathrm{b}}$ Department of Computer Science, University of Copenhagen, Universitetsparken 1, DK-2100 Copenhagen, Denmark
}

Available online 14 November 2006

\begin{abstract}
Animating physically realistic human characters is challenging, since human observers are highly tuned to recognize human cues such as emotion and gender from motion patterns. The main contribution of this paper is a new model firmly based on biomechanics, which is used to animate balance and basic movements of a ballet dancers. It is supported by computer simulated experiments and it is in good agreement with biomechanical measurements of real-life dancers. Our results questions the previous approaches in dynamic animation, which only uses the center of gravity strategy, and instead demonstrate the viability of the center of pressure strategy.
\end{abstract}

(C) 2006 Elsevier B.V. All rights reserved.

Keywords: Biomechanics; Balance strategy; Weight shift strategy; Control mechanism

\section{Introduction}

A long term goal of computer graphics is to increase realism and believability in computer generated animations and pictures $[1,23,12,4,28,3]$. With improved rendering techniques, the lack of physical realism and believability is becoming increasingly obvious and annoying to the common observer. One accompanying long term goal in animation is to increase physical realism by using physics to model plausible behavior and movement of computer models. This known as physics-based animation.

This paper studies biomechanical and ballet inspired balance and weight shifting strategies and is based on original work in [27]. Ballet is a balance art and is a prime focus for learning about human balance and weight shifting strategies. We will describe how to make a dynamic animation from the first of the basic positions in Fig. 1 to obtain a quiet standing on one toe for a 3D humanoid model. To achieve the goal, the articulated figure moves through the four sub-goals shown in Fig. 2: Balancing on two legs, weight shift to the supporting leg, balancing on one leg, and balancing on the toe of one leg. The new model is firmly based on biomechanics and is supported by computer simulated experiments showing good agreement with biomechanical measurements of real-life dancers.

\footnotetext{
${ }^{*}$ Corresponding author.

E-mail address: sporring@diku.dk (J. Sporring).
} 


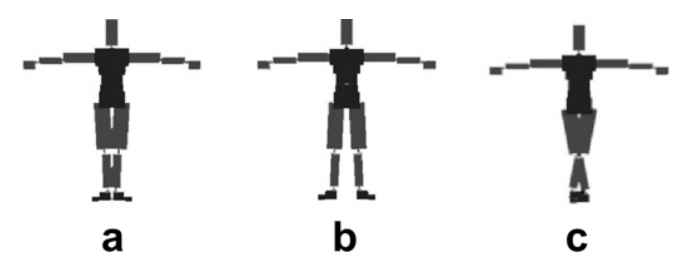

Fig. 1. Three of the basis ballet positions shown in our simulator: (a) First, (b) Second, and (c) Fifth. The feet are positioned in the frontal plane in all poses.

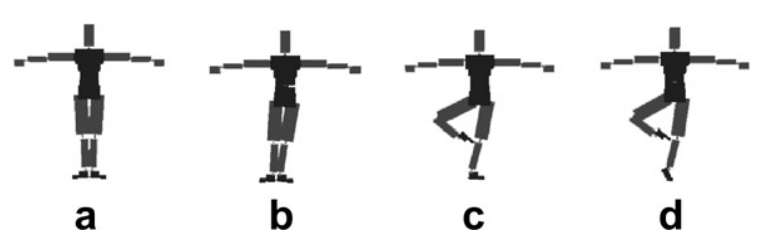

Fig. 2. Images from our simulator illustrating shifting the weight from both legs in first pose (a) to the left leg (b), to a one-legged stand (c), and finally to a one-toed (d) quiet standing.

\subsection{Survey of past work}

The study of balance has been performed in at least three separate areas of research: Biomechanics, Robotics, and Animation. We will in the following highlight some research from these areas.

Biomechanics and the study of ballet: Ballet is an art-form, where balance plays a central role. Classical ballet techniques are throughly described in the literature see, e.g. [11,31]. Biomechanical studies of ballet has mainly been studied through injury cases, e.g. [9]. In the studying of human balance there are two theories: Either we balance by controlling the center of mass directly or indirectly by controlling the center of pressure [20]. Empirical investigations have shown that the velocity of center of mass plays a role in balancing [32]. Empirical studies on real humans have been performed on balancing of humans versus the position of the center of mass [26], and shifts have been measured using a force-platform [24,25]. Finally, a thorough measurement of the properties of mass and inertia of human body parts may be found in [7].

Robotics: Early work on balancing robots may be found in [30]. Weight shift strategies for walking are often performed through dynamic walking machines, where there is more or less degree of control involved in the walking cycle $[21,17,29]$. Recently, a number of spectacular and stable humanoid robots have been produced starting with the Honda robot [15]. Especially in relation to our work, the center of pressure has recently been introduced as a stable control mechanism for balancing robots $[14,13]$. However spectacular complex motions, such as shown in Fig. 2 has not been attempted within these robots.

Animation: A very early mentioning of simulated human motion is given in [2], and early implementations may be found in [10,22]. The implementation of dancing models is only scarcely discussed in the literature, however one exception is [6]. A major inspiration for our work has been [18,33], where center of mass is used to control the balance and motion of a humanoid model of varying complexity. Alternative to center of mass strategies are inverse kinematics, e.g. [19], energy models, e.g. [5], and learning approaches, e.g. [8].

\section{The biomechanics of quiet standing}

A human in quiet standing may be modeled by an articulated figure [18,33] consisting of a set of joints and a set of links representing body parts. The set of possible joints consist of revolute ( 1 degr of freedom (DOF)), universal (2 DOF), and ball-and-socket (3 DOF) joints. In this work, we have used Wooten's model [33], which contains 28 DOFs, and uses real measurements of the mass, $m_{i}$, center of mass, $\vec{r}_{i}$ and moments of inertia of all the body parts [7]. The ankle and hip joints is of particular importance for this paper, and they are modeled by an universal joint and a ball-and-socket joint respectively.

Traditionally in biomechanics and anatomy motion orientation is described in three planes: The Sagittal ( $x$-axis), the transverse ( $y$-axis) and the frontal (z-axis) plane. These planes are illustrated in Fig. 3(a). 


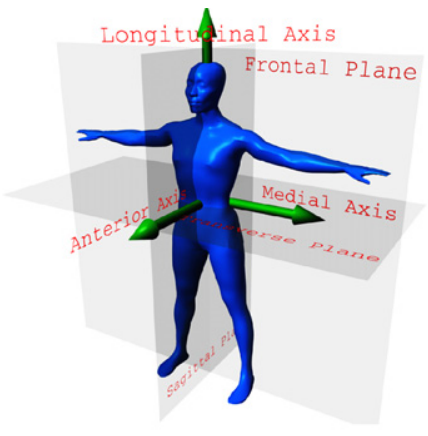

a

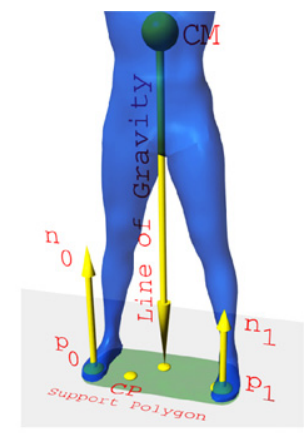

b

Fig. 3. (a) Sagittal, transverse, and frontal planes together with medial, longitudinal and anterior axis. (b) Illustration of biomechanical definitions: Center of mass, center of pressure, support polygon and line of gravity.

Measurements of angles and positions are traditionally also performed in these planes [24] by projection onto the respective planes and axes. Typical projections are: The position of the center of mass, the position of the center of pressure, angle of joints, and the direction of gravity. Working with the projections, rather than the underlying 3D geometry, allows for comparison with the substantial biomechanical literature.

Muscles are used to move and sustain posture of the human skeleton, and our articulated figure is supplemented by an actuator system, which applies joint torques according to a simple damped angular spring model,

$$
\tau=k_{s}^{\text {muscle }}\left(\theta^{\text {target }}-\theta^{\text {current }}\right)-k_{d}^{\text {muscle }} \dot{\theta}^{\text {current }} .
$$

In the equation, $\tau$ is the length of the torque vector, $\theta^{\text {target }}$ and $\theta^{\text {current }}$ are target and current angles, $\dot{\theta}^{\text {current }}$ is the current velocity of the angle, and $k_{\mathrm{s}}^{\text {muscle }}$ and $k_{\mathrm{d}}^{\text {muscle }}$ are spring and damping constants.

A balance control strategy is a function that determine updates, $\Delta \theta$ 's, based on the current state of the articulated figure, $\theta^{\text {current }}$ 's, such that the model will converge towards a desired state of quiet balance, i.e. the strategy iteratively determines new parameter values, $\theta^{\text {new's }}$ as,

$$
\theta^{\text {new }}=\theta^{\text {current }}+\Delta \theta \text {. }
$$

In the rest of this article, it will be assumed that the model is placed on a planar floor, and the contact between the floor and the feet is represented by a set of coplanar contact points, $\vec{p}_{j}$. The support polygon is defined as the $2 \mathrm{D}$ convex hull of all the contact points. The center of mass of the human model is defined as,

$$
\vec{r}_{\mathrm{cm}}=\frac{1}{M} \sum_{i}^{N} m_{i} \vec{r}_{i}
$$

where $M=\sum_{i}^{N} m_{i}$ is the total mass, $N$ is the number of body parts in the model, $m_{i}$ is the individual weights of the body parts, and $r_{i}$ are their locations. Note that center of mass is not fixed w.r.t. any location of the body during motion of the individual body parts. The center of pressure is defined as,

$$
\vec{r}_{\mathrm{cp}}=\frac{1}{\|\vec{n}\|} \sum_{j}^{K}\left\|\vec{n}_{j}\right\| \vec{p}_{j},
$$

where $\vec{n}=\sum_{j}^{K} \vec{n}_{j}$ is the total normal force acting on the human model, $\vec{n}_{j}$ is the normal force applied to the human model at the $j$ th contact point $\vec{p}_{j}$ and $K$ is the number of contact points. is,

For simplicity, a spring model for the floor contact forces is used, where the contact force of the $j$ th contact

$$
\vec{f}_{j}=k_{\mathrm{s}}^{\text {contact }}\left(\vec{p}_{j}^{\text {initial }}-\vec{p}_{j}\right)-k_{d}^{\text {contact }} \dot{\vec{p}}_{j}
$$

In the equation, $\vec{p}_{j}^{\text {initial }}$ is the initial point of contact, $\vec{p}_{j}$ is the current contact point, $\dot{\vec{p}}_{j}$ is the velocity of the current contact point, and $k_{\mathrm{s}}^{\text {contact }}$ and $k_{\mathrm{d}}^{\text {contact }}$ are spring and damping constants. The vector, $\vec{n}_{j}$, is calculated 
as the projection of $\vec{f}_{j}$ onto the contact normal of the floor, and the tangential part is a simple model of fractional force.

Contact forces can only be repulsive, and attractive contact forces, $f_{j}$, are therefore set to zero. Slipping is obtained by setting $\vec{p}_{j}^{\text {initial }}$ equal to $\vec{p}_{j}$ when the magnitude of the tangential force component exceeds a multiple of the magnitude of the normal force component, $\left\|\vec{f}_{\text {friction }}\right\|>-\mu\|\vec{n}\|$.

The line of gravity is defined as the line going from the center of mass to the ground in the direction of the gravitational field. The point of intersection between the line of gravity and the floor is referred to as the projection of the center of mass. These concepts are illustrated in Fig. 3(b).

Balance is defined as an objects ability to maintain quiet standing, where quiet standing is obtained when the projection of the center of mass is kept within the support polygon $[16,18,33]$. The implication is that the greater support polygon, the lower center of mass, the more stable the balance and vice versa. The human body has a highly placed center of mass over a rather small support polygon, and as such the human body behaves as an inverted pendulum.

In the remainder of this paper, control strategies for maintaining quiet standing and moving an articulated figure from one pose of quiet standing to another will be discussed.

\section{Balance strategies: mass center versus pressure center}

In the following, two strategies for balance inspired by biomechanics will be compared: The center of mass strategy and the center of pressure strategy. The analysis are done on an articulated figure in 3D based on [33] standing with parallel. The balance is controlled by the ankle [32].

The center of mass strategy is the traditional balance strategy for dynamic animation, where the angular change is controlled as a function of the projection of the center of mass onto the support plane $[18,33]$ :

$$
\Delta \theta=k_{\mathrm{s}}^{\mathrm{cm}}\left(r_{\mathrm{cm}}^{\mathrm{current}}-r_{\mathrm{cm}}^{\mathrm{target}}\right)-k_{\mathrm{d}}^{\mathrm{cm}} v_{\mathrm{cm}},
$$

where $\Delta \theta$ is the angular changes of the ankle, $r_{\mathrm{cm}}^{\text {current }}$ and $r_{\mathrm{cm}}^{\text {target }}$ are the projections of the current and target positions of the center of mass onto the transverse plane, $v_{\mathrm{cm}}$ is the velocity vector of the center of mass and $k_{\mathrm{s}}^{\mathrm{cm}}$ and $k_{\mathrm{d}}^{\mathrm{cm}}$ are control parameters.

In the center of pressure strategy, the model uses the center of pressure to control the center of mass. The goal of this strategy is to calculate a desired position of the center of pressure, and use this for calculating the muscles control:

$$
\begin{aligned}
& \Delta r_{\mathrm{cp}}=k_{\mathrm{s}}^{\mathrm{cm}}\left(r_{\mathrm{cm}}^{\mathrm{current}}-r_{\mathrm{cm}}^{\mathrm{target}}\right)-k_{\mathrm{d}}^{\mathrm{cm}} v_{\mathrm{cm}}, \\
& r_{\mathrm{cp}}^{\mathrm{target}}=\Delta r_{\mathrm{cp}}+r_{\mathrm{cm}}^{\mathrm{current}}, \\
& \Delta \theta=k_{\mathrm{s}}^{\mathrm{cp}}\left(r_{\mathrm{cp}}^{\mathrm{current}}-r_{\mathrm{cp}}^{\mathrm{target}}\right)-k_{\mathrm{d}}^{\mathrm{cp}} v_{\mathrm{cp}},
\end{aligned}
$$

where $\Delta r_{\mathrm{cp}}$ is the positional change of the center of pressure, $r_{\mathrm{cp}}^{\text {current }}$ and $r_{\mathrm{cp}}^{\text {target }}$ are the current and the target positions of the center of pressure, and $k_{\mathrm{s}}^{\mathrm{cp}}$ and $k_{\mathrm{d}}^{\mathrm{cp}}$ are control parameters.

The center of pressure theory can be understood by looking at the human balance like the balance of an inverted pendulum. The center of mass is the top and on the floor is the center of pressure. When the positions are right above each other, it is a perfect balance. In order to change the balance to a new desired position. The center of mass can start an acceleration towards the new point by moving the center of pressure in the opposite direction to initiate a falling motion. You do the same when you balance a match on your finger.

Fig. 4 shows the resulting dynamics of the projected center of mass and the center of pressure on the anterior axis. The movement of the projection of the center of mass on the articulated figure start in a position on the heels to the desired position on the center of the feet (the center of the support polygon). The articulated figure manages to balance using both strategies, but the center of pressure strategy requires only approximately $1.5 \mathrm{~s}$ while the center of mass strategy requires almost $10 \mathrm{~s}$ to get a balance in the desired position. In addition, the center of pressure strategy also has the smallest amplitude of the oscillations of the center of pressure. This means that it has the best control over the contact with the ground, because movements of the center of pressure means that the weight on the feed are changing. The articulated figure in the balance tests has only 1 DOF joints and is therefore only balancing by the equations in the sagittal plane. This is fine 

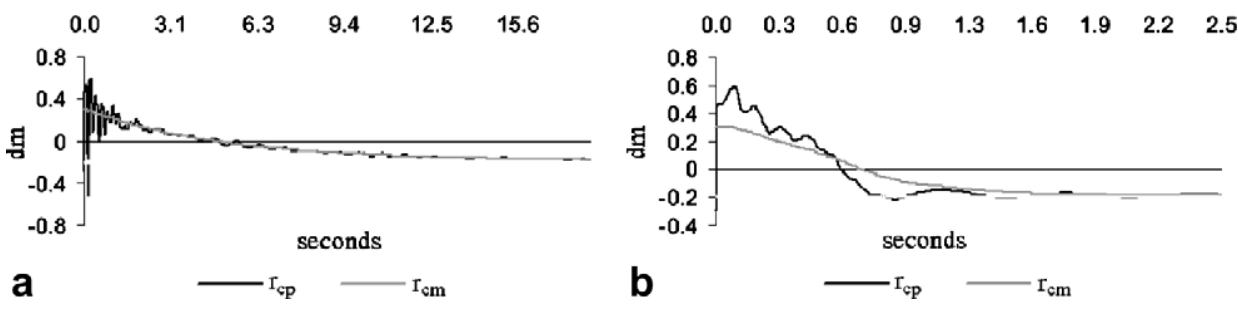

Fig. 4. Comparison of the dynamics of the ankle for the two strategies: (a) Center of mass strategy and (b) Center of pressure strategy. $r_{\mathrm{cp}}$ is the center of pressure and $r_{\mathrm{cm}}$ is the center of mass.

because the position of the projection of the center of mass is the same as the position of the center of pressure on the medial axis from start. In all our other tests the articulated figure is both balancing in sagittal and in frontal plane. We have done the balance tests on another model [27] than Wooten's [33] and obtained similar results.

\section{Dynamics of a ballet dancer}

Quiet standing on the toe of one leg is central in all ballet training. This is demanding, since the dancer has to balance on a very small support polygon while at the same time looking at ease. We have developed strategies for obtaining a balance on the left leg with the right foot by the left knee as illustrated in Fig. 2. The motion to balance is obtained through a number of balance controllers and muscle actions shown in Fig. 5(a), and the details of which will be discussed in the following.

The one leg pose is used, when dancers turn in a pirouette, and it is perhaps the most basic of all balances in ballet training. Both in quiet balance and in most of the basic ballet exercises, the legs are strictly separated into the working leg (doing the exercise) and the supporting leg. Shifting the weight between the legs is basic, and it should preferably be done without drawing the attention of the audience. In our tests the left leg is the supporting leg, and the right leg is the working leg.

\subsection{Weight shifting strategies}

The weight shifting strategy described in the following was inspired by the analysis of real dancers presented in the literature [24]. They showed that angle changes in the hips and ankles are nearly identical during a weight shift, and that the center of pressure starts moving towards the working leg and end up being on the supporting leg. The last result is only explainable from the center of pressure balance strategy.

To shift the weight, we designated the left ankle to be the controlling joint using (9). The desired position of the projection of the center of mass where on the center of the supporting legs foot. The angular changes in the hip joints where calculated from the angular changes in the left ankle. The right ankle controlled the position of the right foots center of pressure.

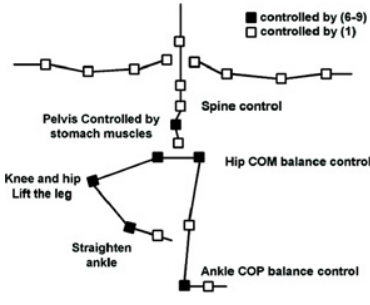

a

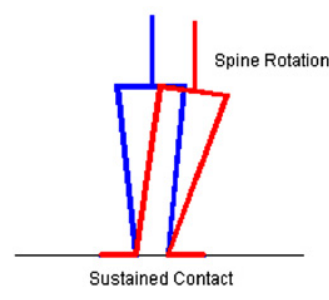

b

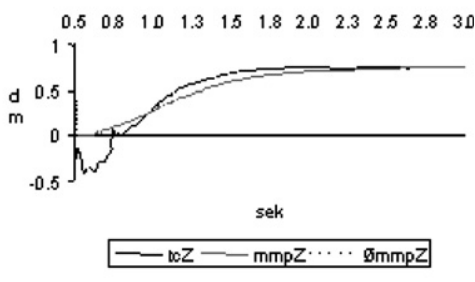

C

Fig. 5. (a) The model, motions, and controllers used to obtained balance on one leg. (b) A rotation in the ankles with straight legs implies a rotation in the hip, and a rotation in the spine is required in order to keep the upper body vertical. (c) Plot showing position of the center of pressure and center of mass projected onto the medial axis. 
Ballet aesthetics requires that the upper body is kept parallel to the line of gravity during a weight shift in the frontal plane with both feet fixed on the floor. It has been claimed [24] that dancers keep their upper body vertical by a counter rotation in the hip joints, however this is only physically possible when the legs are parallel as illustrated in Fig. 5(b). Ballet dancers compensate for the hip rotation by a counter rotation in the lumbar region of the lower back [11], and therefore a control function in the spine is required. Dancers control the body center by the stomach muscles. Experience has shown that these stomach muscles are extremely important for aesthetic motion of the articulated figure [27], thus a control function in the pelvis is used to inhibit rotation in the Sagittal plane. Both control functions are modeled using a spring law similar to (1).

The final weight shift of the articulated figure is shown in Fig. 2. The resulting articulated figure agrees with measurements performed on real dancers [24] as follows: The measured angle changes are nearly identical during the weight shift, and the center of pressure starts to move towards the right foot and ends in a position on the left foot. The last result are showed in Fig. 5(c). On the din-axis zero is between the center of the legs, the negative numbers are the foot of the working/right leg and the positive are foot of the supporting/left leg.

\subsection{Movement to quiet standing on one toe}

The final movements to obtain a one-toed quiet standing is achieved by lifting the non-supporting leg, and shifting from a foot stand to a toe stand, see Fig. 2. It is not difficult to raise the leg, however the major challenge is to keep balance on a very small support polygon.

Two strategies have been developed: A strategy for raising the right leg, and a strategy for making a weight shift to the toe. Similarly to the weight shifting balance strategy, both are based on the center of pressure strategy.

To raise the leg, the left ankle is kept as the controlling joint, and the center of pressure strategy is used to keep the balance on the center of the left foot. To shift the weight to one toe, the center of pressure strategy is used in two steps: Firstly, for the controlling ankle joint, and secondly to control the toe joint, when the ankle has been straightened.

When the support polygon is getting smaller the human body's small deviations from a stiff pendulum makes it necessary to have a control of the center of mass at the left hip joint to maintain the balance.

Lifting the right leg to an aesthetically pleasing pose is performed using a spring law. The spine and pelvis are controlled as explained in the previous section.

The analysis of the results shows a motion of the center of pressure, when the right foot let go of the floor contact. This is not entirely in agreement with measurements on real dances. Otherwise the result shows a stable line.

\section{Discussion}

From a ballet point of view, controlling the center of pressure is also a way of controlling the contact of the feet with the ground. Ballet dancers are very much aware of the relation between their feet and the ground, since it strongly influences their balance, their stance, and the audiences impression of the dancers body. Balance and weight shifting are the most basic techniques in ballet.

This paper has shown how you can use results from biomechanics for developing strategies for dynamic animations of an articulated figure of a human. The focus has been on balancing strategies, where two strategies have been compared: center of mass strategy, and the center of pressure strategy. The latter showed far the best results, and our results demonstrated that using the center of pressure strategy makes it possible to develop strategies for an articulated figure of a human to balance in a very complicated position.

The center of pressure strategy is connected with the theory of the balance of the inverted pendulum. We have used it in a simple form just working on the ankle joint, and we demonstrated that it is an effective way of balancing for quiet standing among other positions. However, the inverted pendulum is a too coarse simplification of the human body, when the working leg is lifted balance is obtained on one leg. This movement requires motion of joints in the whole body. We had to rely on the center of mass strategy at the hip joint to stabilize the balance on one leg. The conclusion is that human balance is complex, and that humans possibly use both the center of pressure strategy and the center of mass strategy. 
The center of pressure strategy as presented in this article is for fine tuning the balance. That is, the strategy is only useful, when the projection of the center of mass is inside the support polygon: In the case of a fall, the natural human response would be to produce counter movements such as moving a leg, which in fact moves and increases the size of the support polygon in order to regain balance; the pressure strategy could be used to estimate useful support polygons in order to calculate a useful leg movement, but such algorithms have not been investigated in this paper.

Our results are in good agreements with results from biomechanics. We attribute the minor deviations observed as simplifications in our model such as the simplified skeleton and the muscles used.

From a designer point of view, the use of virtual spring for modeling actuator forces and contact forces are attractive due to their simplicity, but they require a lot of parameter tuning, and these parameters are rather sensitive to global model changes. Learning may be incorporated to avoid excessive parameter tuning, but result are not guarantied to look aesthetically pleasing. Research in finding a better method for finding parameters could make physical animation of humans much more useful also in areas outside research. Future steps in our research will be to develop strategies for exercises on one leg.

\section{References}

[1] William W. Armstrong, Mark W. Green, The dynamics of articulated rigid bodies for purposes of animation, The Visual Comput. 1 (4) (1985) 231-240.

[2] Norman I. Badler, Stephen W. Smoliar, Digital representations of human movement, ACM Comput. Surv. 11 (1) (1979) 19-38.

[3] B.A. Barsky, N. Badler, D. Zeltzer (Eds.), Making Them Move: Mechanics Control and Animation of Articulated Figures, The Morgan Kaufmann Series in Computer Graphics and Geometric Modeling, Morgan Kaufman Publishers Inc, 1991.

[4] R. Barzel, A.H. Barr, A modeling system based on dynamic constraints, In Comput. Graph. 22 (1988) $179-187$.

[5] Ronan Boulic, Ramon Mas-Sanso, Daniel Thalmann, Complex character positioning based on a compatible flow model of multiple supports, IEEE Trans. Vis. Comput. Graph. 3 (3) (1997).

[6] Tom Calvert, Composition of realistic animation sequences for multiple human figures, in Barsky et al. [3], chapter 2.

[7] Wilfred Taylor Dempster, George R.L. Gaughran, Properties of body segments based on size and weight, Am. J. Anat. (1967).

[8] Petros Faloutsos, Michiel van de Panne, Demetri Terzopoulos, Composable controllers fro physics-based character animation, in Eugene Fiume (Ed.), SIGGRAPH 2001, Computer Graphics Proceedings, ACM Press/ACM SIGGRAPH, 2001, pp. 251-260.

[9] Donald F. Featherstone, Dancing Without Danger, Kaye and Ward Limited, England, 1970.

[10] M. Girard, A.A. Maciejewski. Computational modeling for the computer animation of legged figures, in: ACM Siggraph 1985, pp. 263-270.

[11] Valerie Grieg, Inside Ballet Technique, Dance Books, London, UK, 1994.

[12] J.K. Hahn, Realistic animation of rigid bodies, In Comput. Graph. 22 (1988) 299-308.

[13] Satoshi ITO, Hironori ASANO, Haruhisa KAWASAKI, A balance control in biped double support phase based on center of pressure of ground reaction forces, in: The 7th IFAC Symposium on Robot Control, vol. 1, Wroclaw, 2003, pp. 205-210.

[14] Satoshi Ito, Haruhisa Kawasaki, A standing posture control based on ground reaction force, in: Proceedings of the 2000 IEEE/RSJ International Conference on Intelligent Robots and Systems, Takamatsu, 2000, pp. 1340-1345.

[15] Fumio Kanehiro, Kenji Kaneko, Kiyoshi Fujiwara, Kensuke Harada, Shuuji Kajita, Kazuhito Yokoi, Hirohisa Hirukawa, Kazuhiko Akachi, Takakatsu Isozumi, The first humanoid robot that has the same size s a human and that can lie down and get up, in: Proceedings of the 2003 IEEE International Conference on Robotics \& Automation, Taipei, Taiwan, September 2003, pp. 1633-1639.

[16] Ellen Kreighbaum, Katharine M. Barthels, Biomechanics, a qualitative approach for studying human movement, fourth ed., Allyn and Bacon, Toronto, Canada, 1996.

[17] Andrew L. Kun, W. Thomas Miller, Adaptive dynamic balance of a biped robot using neural networks, in: Proceedings IEEE International Conference on RA, 1996, pp. 240-245.

[18] Joseph Laszlo, Controlling bipedal locomotion for computer animation. Master's thesis, University of Toronto, Canada, 1996.

[19] Joseph Laszlo, Michiel van de Panne, Eugene Fiume, Limit cycle control and its application to the animation of balancing and walking, in: Proceedings of SIGGRAPH 1996, New Orleans, LA, 1996, pp. 155-162.

[20] Column D. Mackinnon, David Winter, Control of whole body balance in the frontal plane during human walking, J. Biomech. 26 (6) (1998) 633-644.

[21] Tad McGeer, Passive dynamic walking, The Int. J. Robot. Res. 9 (2) (1990) 62-82.

[22] Michael McKenna, David Zeltzer, Dynamic simulation of autonomous legged locomotion, Comput. Graph. 24 (4) (1990) 29-38.

[23] M. Moore, J. Wilhelms, Collision detection and response for computer animation, Comput. Graph. 22 (1988) $289-298$.

[24] L. Mouchnino, R. Aurenty, J. Massion, A. Pedotti, Coordination between equilibrium and head-trunk orientation during leg movement: A new strategy built up by training, J. Neurophysiol. 67 (6) (1992).

[25] E. Otten, Balancing on a narrow ridge: biomechanics and control, Philos. Trans.: Biolog. Sci. 354 (1385) (1999) 69-875.

[26] Yi-Chung Pai, James Patton, Center of mass velocity-position predictions for balance control, J. Biomech. 30 (4) (1997) $347-354$.

[27] Camilla Pedersen, Balancekontrol og strategier til en dynamisk animation af en balletdanser [balance control and strategies for a dynamical animation of a ballet dancer]. Master's thesis, IT University of Copenhagen, Denmark, 2002. 
[28] J.C. Platt, A.H. Barr, Constraint methods for flexible bodies, Comput. Graph. 22 (1988) 279-288.

[29] Jerry E. Pratt, Gill A. Pratt, Exploiting natural dynamics in the control of a 3D bipedal walking simulation, in: International Conference on Climbin and Walking Robots (CLAWAR9i9), Portsmouth, UK, 1999.

[30] M.H. Raibert, Legged Robots That Balance, MIT Press, Cambridge, MA, 1986.

[31] Gretchen Warren, Classical Ballet Technique, University Press of Florida, 1989.

[32] David Winter, Aftab Patla, Francois Prince, Milad Ishac, Krystyna Gielo-Perczak, Stiffness control of balance in quiet standing, J. Neurophysiol. 80 (3) (1998) 1211-1221.

[33] Wayne L. Wooten, Simulation of Leaping, Tumbling, Landing, and Balancing Humans, PhD thesis, Georgia Institute of Technology, USA, 1998. 